\title{
UKRN Position on Supporting Research Transparency among Research Students
}

The UK Reproducibility Network Steering Group $27^{\text {th }}$ July 2021

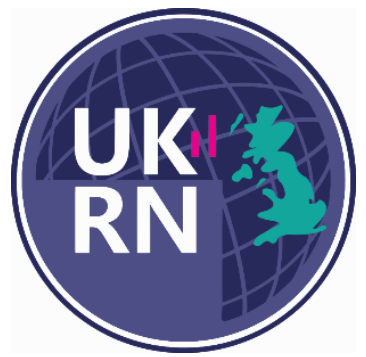

The UK Reproducibility Network (UKRN) is committed to promoting transparency and rigour in research across all disciplines and to improving the ways in which we conduct research. Making the research process as open as possible is a core part of research transparency, and open research practices are now explicitly rewarded at many UKRN stakeholder institutions, for example in promotion decisions and Open Research Prizes.

We particularly recognise the importance of developing transparent research practices from the earliest stages of a research career, and encourage institutions to teach, promote, and reward transparency among research students.

\section{Transparency across disciplines}

We also recognise that transparency in research and scholarship varies considerably across disciplines and methodologies. Therefore, research students should be encouraged and supported to pursue transparency through the most appropriate and effective means according to the nature of their research. The transparency of research methods - being open about what was done, how, and why - is essential in all research contexts, to allow the research community to form a solid foundation upon which to build further knowledge.

In those research contexts where sharing of data is impossible or impracticable (e.g., due to privacy concerns, or the interpretative nature of the research), we would still encourage research students to strive for maximum transparency. Depending on the discipline and context, transparency can be demonstrated by, for example, the use of project websites to explain the researchers' decisions or intermediate results - so that future researchers can fully understand how to build on this work.

In contexts where reproducibility of the results is possible and appropriate (i.e., using the same or similar data and/or methods to produce the same/similar results), we strongly encourage research students to use measures such as sharing data sets, code and materials on institutional repositories or appropriate external platforms. Preparing such materials can help students communicate their procedures with their thesis advisors, examiners, and beyond.

As well as supporting research reproducibility, these practices foster the wider and more rapid dissemination, discovery and re-use of research outputs, which is of particular benefit to early career researchers.

\section{Publication of theses and work therein}

Students who submit a thesis as all or part of their $\mathrm{PhD}$ evaluation should post the thesis on a dedicated repository (e.g., an institutional repository, or the EThOS platform) and make it publicly available as soon as is practical. 
Where students have submitted work included in their thesis for publication (e.g., where work described in a PhD chapter has been published in a journal), this work should be evaluated on its own merits in the context of the evaluation of the thesis, irrespective of where or how it has been published.

One implication of this is that thesis work should not be evaluated with reference to inappropriate metrics that unevenly favour particular disciplines or are affected by time elapsed since publication (e.g., number of citations, or Journal Impact Factor).

Ultimately, we encourage institutions and examiners to provide training in open research practices, and to recognise and reward work that reflects this approach. By doing so, institutions will demonstrate their commitment to transparency in research, and instil these ways of working from the outset of the careers of the next generation of researchers.

\section{(c) (1) (1)}

This work is licensed under a Creative Commons Attribution-ShareAlike 4.0 International License. 